\title{
Think Heterotopic: A Case Report of Heterotopic Pregnancy Detected on Thorough Ultrasonography
}

\author{
Tng Chang Kwok ${ }^{\mathrm{a}, \mathrm{b}}$, George Morgan ${ }^{\mathrm{a}}$
}

\begin{abstract}
We present a case report of heterotopic pregnancy picked up on thorough ultrasonography in a clinically stable 22-year-old patient with low risk factors. Heterotopic pregnancy is a rare obstetrics phenomenon and carries a significant maternal morbidity and mortality due to the risk of rupture of the ectopic pregnancy. Clinicians and sonographers tend to be lulled into a false sense of security when an intrauterine gestation sac is seen. This results in inadequate inspection of the adnexae and remaining structures during ultrasonography despite a strong initial clinical suspicion of ectopic pregnancy. Hence, a thorough ultrasonography and holistic approach is needed in managing these patients, especially when there is a high suspicion of ectopic pregnancy or in the presence of pelvic free fluid even when an intrauterine pregnancy is identified. A new dictum 'Think ectopic. If intrauterine gestation is seen, think heterotopic' needs to be adopted.
\end{abstract}

Keywords: Heterotopic pregnancy; Ultrasonography; Ectopic; Holistic

\section{Introduction}

Heterotopic pregnancy is defined as the simultaneous gestation in 2 or more implantation sites. First found during an autopsy in 1708 [1], heterotopic pregnancy occurs in 1:30,000 of natural conceptions [2]. With increasing usage of assisted reproduction, the frequency of heterotopic pregnancies was cited to be between 1:100 and 1:7,000 [3,4].

This rare obstetrics phenomenon carries a significant maternal morbidity and mortality due to the risk of ruptured

\footnotetext{
Manuscript accepted for publication July 10, 2012

${ }^{a}$ Obstetrics and Gynaecology Department, Kings Mill Hospital, Sutton in Ashfield, Nottinghamshire, NG17 4JL, United Kingdom

${ }^{\mathrm{b}}$ Corresponding author: Tng Chang Kwok. Email: tkwok@nhs.net
}

doi:10.4021/jmc779w ectopic pregnancy [5]. Hence, early diagnosis and management of heterotopic pregnancy is crucial. However, the rate of early diagnosis of heterotopic pregnancy has not increased since 1970 despite advances in medical knowledge and ultrasonography technique [6].

Health care professionals have been indoctrinated with the famous dictum by the French surgeon Henri Mondor (1885 - 1962) 'Think Ectopic' when assessing acute abdomen of pregnant women. However, when an intrauterine gestation is seen on ultrasonography, the dictum is not translated into heterotopic pregnancy.

Below is a unique case history of heterotopic pregnancy in a clinically stable patient with low risk factors which was picked up on thorough ultrasonography rather than on laparoscopy. This case report stresses the importance of a holistic approach and thorough ultrasonography in managing these patients.

\section{Case Report}

$\mathrm{KH}$, a 22-year-old woman, presented to the gynaecological admission unit with a 7-week history of amenorrhea and a 2-day history of lower abdominal period-like pain. She denied any vaginal bleeding, shoulder tip pain, urinary or

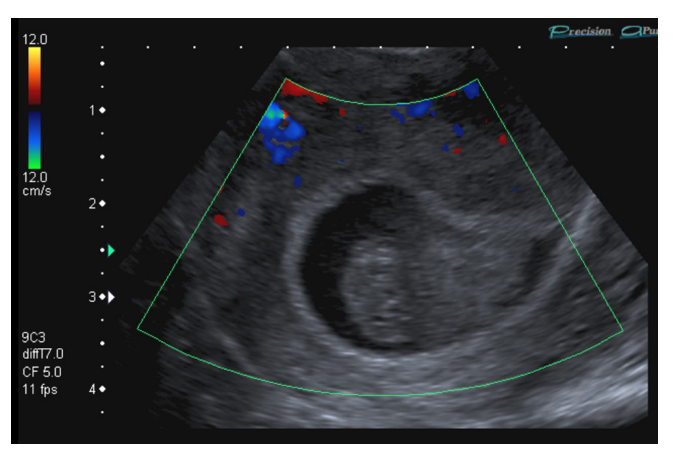

Figure 1. Transvaginal ultrasound scan showing non-viable intrauterine gestation with probable fetal pole without yolk sac nor fetal cardiac pulsation. Crown lump length was estimated at $11 \mathrm{~mm}$ (7+ weeks gestation). Visualisation of content of gestational sac is hard due to haemorrhagic material. 


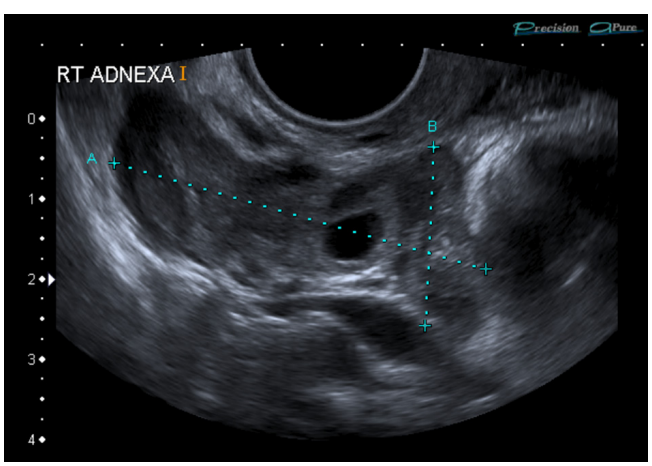

Figure 2. Transvaginal ultrasound scan showing a large irregular heterogenous mass of $48 \mathrm{~mm} \times 31 \mathrm{~mm} \times 21 \mathrm{~mm}$ with $12 \mathrm{~mm} \times 6 \mathrm{~mm} \times 5 \mathrm{~mm}$ probable yolk sac consistent with right ectopic pregnancy.

bowel symptoms.

She denied having had any previous sexually transmitted infections and had never used intra-uterine contraceptive devices or undergone fertility treatment. She had no history of any tubal or pelvic pathology, ectopic pregnancies or miscarriage. She was an ex-smoker. She was on cerazette for contraception which she said she took regularly. Her periods were irregular. She had a normal vaginal delivery in 2008.

Her past medical and surgical histories were unremarkable. She used no regular medications. She was allergic to penicillin.

On examination, she was tachycardic at 105 beats per minute with a blood pressure of $122 \mathrm{mmHg} / 62 \mathrm{mmHg}$. Her abdomen was soft with mild suprapubic and right iliac fossa tenderness. On bimanual examination, she had an 8-week size uterus with a tender right adnexa and positive cervical excitation tenderness. No adnexal masses were felt. Her cervical os was closed.

Her urine pregnancy test was positive. Her serum bHCG was $3892 \mathrm{mIU} / \mathrm{mL}$. Urine dipstick showed no signs of urinary tract infection. Her haemoglobin was $13.7 \mathrm{~g} / \mathrm{dL}$.

She underwent a transabdominal and transvaginal ultrasound scan which revealed a non-viable intrauterine gestational sac at 7+ weeks (Fig. 1) as well as a large heterogenous mass with a yolk sac seen medial to the right ovary (Fig. 2). Free fluid was noted in the pouch of Douglas (Fig. 3).

A laparoscopic right partial salpingectomy and evacuation of retained product of conception was urgently performed which confirmed a right ampullary ectopic pregnancy. The serum bHCG after 48 hours fell optimally to 44.1 $\mathrm{mIU} / \mathrm{m}$. Urinary pregnancy test was negative after 2 weeks.

\section{Discussion}

Early diagnosis of heterotopic pregnancy is often challenging due to the lack of clinical signs and symptoms as well as

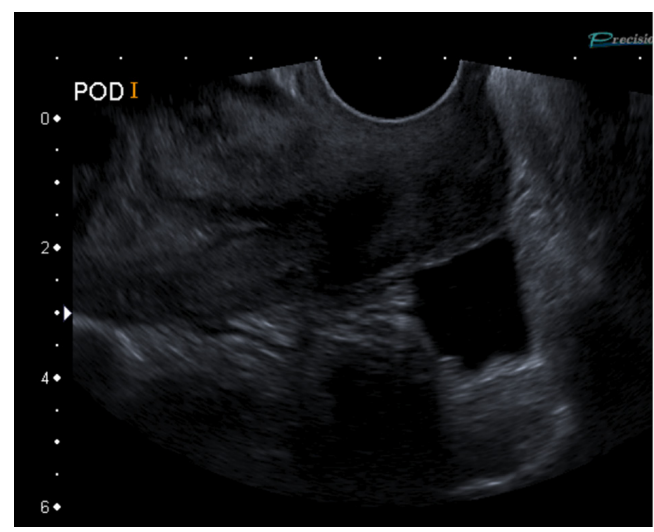

Figure 3. Transvaginal ultrasound scan showing free fluid in the pouch of Douglas indicated by increased echogenicity.

diagnostic confusion with other early pregnancy issues [3].

A good history is important to identify risk factors for heterotopic pregnancy such as fertility treatment and tubal pathologies like pelvic inflammatory disease, endometriosis or previous tubal surgeries [4]. Patients with previous ectopic pregnancy or patients who conceived while using intrauterine devices are also at risk [6].

The four common clinical signs of heterotopic pregnancies are abdominal pain, adnexal mass, peritoneal irritation and uterine fundus larger than menstrual dates [1]. Vaginal bleeding occurs rarely in heterotopic pregnancy, compared to ectopic pregnancy [7].

\section{Ultrasonography}

Ultrasonography is routinely used in early pregnancy. Despite the advancement of transvaginal ultrasonography, identification of viable early intrauterine pregnancy before 5.5 weeks and ectopic pregnancy still remains challenging. Clinicians and sonographers tend to be lulled into a false sense of security when an intrauterine gestation sac is seen. This results in inadequate inspection of the adnexae and remaining structures during ultrasonography despite a strong initial clinical suspicion of ectopic pregnancy.

A review article in 2008 [8] which analysed 6 studies showed that transvaginal ultrasonography has a sensitivity of $74-84 \%$ in diagnosing ectopic pregnancy with specificity between $84-99.9 \%$. However, ultrasonography is operator dependent. A comparative review of 192 cases of heterotopic pregnancies in 2007 [6] showed that only $1 / 3$ of the cases was definitively diagnosed by ultrasonography pre-operatively like in this case study.

The only pathognomonic sign of heterotopic pregnancy is the simultaneous visualisation of extrauterine and intrauterine fetal pole with cardiac motion. This occurs in only $10 \%$ of cases [9]. The ultrasound appearance of an ectopic pregnancy varies depending on location, gestation period, 
viability and presence of pelvic pathology.

However, there are a few features on ultrasonography associated with ectopic pregnancies. Firstly, the risk of ectopic pregnancy is increased with increasing amount of free fluid in the pelvis [3] due to a physiological response to distortion of the Fallopian tube or bleeding from the damaged ectopic pregnancy. Hence, presence of free fluid in pelvis should trigger a thorough inspection of the adnexae despite observing an intrauterine gestation. We should not too hastily attribute the intraperitoneal free fluid to ovarian hyperstimulation syndrome in patients on fertility treatment [6]. Secondly, it is crucial to visualise the corpus luteum. In $70-85 \%$ of cases, ectopic pregnancy normally occurs on the ipsilateral side of the corpus luteum [8].

Ectopic pregnancy is normally visualised on ultrasound in 3 forms [8]. In $58 \%$ of cases, the 'blob' sign, which is a heterogenous mass adjacent to the ovary but moves separately, is seen, $20 \%$ of cases show up as the 'bagel sign', which is a mass with hyperechoic ring around. The remaining cases present as a gestational sac with fetal pole like in this case study.

\section{Conclusion}

A holistic approach and thorough ultrasonography is crucial in patients with high suspicion of ectopic pregnancy or in the presence of pelvic free fluid even when an intrauterine pregnancy is identified. A new dictum 'Think ectopic. If intrauterine gestation is seen, think heterotopic' needs to be adopted.

\section{Grant Support}

None.

\section{References}

1. Varras M, Akrivis C, Hadjopoulos G, Antoniou N. Heterotopic pregnancy in a natural conception cycle presenting with tubal rupture: a case report and review of the literature. Eur J Obstet Gynecol Reprod Biol. 2003;106(1):79-82.

2. De VR, Pratt JH. Simultaneous intrauterine and extrauterine pregnancy. Am J Obstet Gynecol. 1948;56(6):11191126.

3. Press GM, MartinezA. Heterotopic pregnancy diagnosed by emergency ultrasound. J Emerg Med. 2007;33(1):2527.

4. Mj G, R R. Heterotopic pregnancy in natural conception. J Hum Reprod Sci. 2008;1(1):37-38.

5. Cantwell R, Clutton-Brock T, Cooper G, Dawson A, Drife J, Garrod D, Harper A, et al. Saving Mothers' Lives: Reviewing maternal deaths to make motherhood safer: 2006-2008. The Eighth Report of the Confidential Enquiries into Maternal Deaths in the United Kingdom. BJOG. 2011;118 Suppl 1:1-203.

6. Barrenetxea G, Barinaga-Rementeria L, Lopez de Larruzea A, Agirregoikoa JA, Mandiola M, Carbonero K. Heterotopic pregnancy: two cases and a comparative review. Fertil Steril. 2007;87(2):417 e419-415.

7. Thomsen T, Brown DF, Nadel ES. Abdominal pain in first trimester pregnancy. J Emerg Med. 2003;24(1):5558.

8. Bignardi T, Alhamdan D, Condous G. Is ultrasound the new gold standard for the diagnosis of ectopic pregnancy? Semin Ultrasound CT MR. 2008;29(2):114-120.

9. O'Brien MC, Rutherford T. Misdiagnosis of bilateral ectopic pregnancies: a caveat about operator expertise in the use of transvaginal ultrasound. J Emerg Med. 1993;11(3):275-278. 